\title{
Platelet Aggregometry Curve Type
}

National Cancer Institute

\section{Source}

National Cancer Institute. Platelet Aggregometry Curve Type. NCI Thesaurus. Code

C114210.

The classification of the curve pattern that is formed as a result of platelet aggregation. 